\title{
Comparison on Effects of Different Sampling Methods for the Disinfection of Ventilator Pipes
}

\author{
Yanming $\mathrm{Li}^{1, \mathrm{a}}$, Zhenzhen Liang ${ }^{2, \mathrm{~b}}$ and Aizhong Zhao ${ }^{3, \mathrm{c}, \Delta}$ \\ ${ }^{1,2}$ Nursing college, beihua university, jilin city, jilin province, china \\ ${ }^{3}$ University hospital of beihua university, jilin city, jilin province, china \\ a843789777@qq.com, b645635390@qq.com, 492964574@qq.com
}

\begin{abstract}
Keywords: sample collection, ventilator pipe, disinfection introduct.
Abstract. Different sampling methods were used to collect samples from ventilator pipes after they were sterilized for selecting an optimal sampling method for the disinfection of ventilator pipes. 200 sets of the ventilator pipes used in clinic were randomly divided into group A and B. The pipes in group A were washed by hand and disinfected by the immersion disinfection method with strongly acidic ionized-water; those in group B were washed with automatic cleaning machine and sterilized with an autoclave. After the disinfection, the samples were collected with a shallow-swabbed, lavage and deep-swabbed, respectively, and the collected samples were sent to the microbiology laboratory for bacterial culture to confirm the disinfection. The results showed that qualified rates of the disinfection with the different sampling methods in group A and B were $98.32 \%$ and $100 \%$ for shallow-swabbed method, $98.32 \%$ and $100 \%$ for lavage, and $76.22 \%$ and $92.67 \%$ for deep-swabbed method, respectively. It is indicated that disinfection effects with the different sampling methods for the disinfection of ventilator pipes should be significantly different and the sampling with deepswabbed method can objectively reflect the disinfecting effect of the ventilator pipe.
\end{abstract}

\section{Introduction}

Ventilator is an essential equipment currently used for rescuing critical patients and patients with respiratory failure, and has been widely used in various clinical departments. Ventilator tube pollution is one of important causes of ventilator-associated pneumonia (VAP) [1], and VAP has greatly increased the mortality and the financial burden of patients. Although a disposable ventilator pipe is available in clinic, the non-disposable ventilator pipe made of silicone material is still chosen to be used in many medium and small hospitals due to the expensive price of the disposable ventilator pipe now. Currently, there is no domestic standardization for the management of ventilator pipe system sterilization, but there are many methods used for the sterilization of ventilator pipe system, such as automatic washing machine for cleaning and washing, ethylene oxide disinfection, peracetic acid disinfection, and immersion disinfection methods with strongly acidic ionized water. Standards for the monitoring and evaluation of disinfection effects vary widely, and generally the samples are collected based on relevant methods for sampling proposed by "Technical Specifications for Disinfection Edition 2002" [2], but the results of sampling from the different parts and with different methods are quite different because the special nature, length and thread-like structure of inner wall of ventilator pipes all can affect the disinfection. In this study, three sampling methods were used for the sterilization of ventilator pipe, and their effects were evaluated and compared.

\section{Materials and Methods}

Materials. Ventilator pipes (reuse silicone high-temperature-resistant gel thread pipe) continuously used for $72 \mathrm{~h} 200$ sets, cleaning brush, strong acidic ionized water, pure water, full effect multienzyme detergent, automatic washing machine, air sterilizer, drying cupboard, sterile sampling tubes, sterile saline, disposable syringes, and sterile cotton swab $(10 \mathrm{~cm} / 40 \mathrm{~cm})$. 
Cleaning and Disinfecting Method. The used ventilator pipes were randomly divided into two groups group A and B. In group A, after they were separated, the components of ventilator pipe system were washed with running water, soaked in the multi-enzyme solution at the ration of 1: 270 for 5min, visible stains on their surfaces and inner wall were cleared away with a soft brush, then rinsed with running water, soaked in the strongly acidic ionized water for $30 \mathrm{~min}$, and then rinsed with pure repeatedly for 5-10min and placed in the specified location for drying or dried with an air sterilizer, and finally wrapped with a double-layer aseptic package for use. It should be noted that all items must be soaked in the solutions completely, the lumen of pipes must be filled with the solutions without air bubbles; all drivepipes and bosses of the threaded pipes must be separated, the water-collecting bottle must be removed and fully opened, and the pipes should not be bent and folded. In group B, after they were separated, the components of ventilator pipe system were cleaned and dried with automatic washing machine. Specific procedures: the ventilator threaded pipe to be sterilized was put into a specialized pipe cleaning rack, a cleaning program was select to start the cleaning. Prewash: washed with 1:500 low foaming enzyme; wash: washed with hot water at $93 \square$ for heat sterilization for $1 \mathrm{mim}$, and dried with a drying cupboard [3] at 83-90 $\square$ for 30min; when the sterilizer of automatic cleaning machine indicated the end of cleaning, the threaded pipes and related items were taken out by opening the door of the wash area and with hands wearing sterile gloves, and wrapped with paper-plastic after they were checked to ensure that they were fully dried; the paper-plastic package was sterilized by an autoclave at $132-134 \square$ for $10 \mathrm{~min}$, and after taking out the package, the disinfection date was given clear indication and the retention period was $7 \mathrm{~d}$.

Sampling Method. A part of each ventilator threaded pipe at a length of $\geq 60 \mathrm{~cm}$ was selected as the sampling object and the samples were collected according to the following methods. Method A (shallow-swabbed method): a 10-cm sterile cotton swab dipping sterile saline was used to circularly swab the surface of the inner wall of the pipe lumen at the distal part approximately at the depth of $5 \mathrm{~cm}$, and the swabbed area was not less than $50 \mathrm{~cm}^{2}$; the finger-touched part of cotton swabs was cut out with a sterile scissors and the residual part of the cotton swabs was placed in a sampling tube for test. Method B (lavage): $10 \mathrm{ml}$ sterile saline was taken with a disposable syringe and injected into the lumen of ventilator pipes, the pipe filled the saline was shaken back and forth 10 times, and then the injected saline was poured into a sterile sampling tube for test. Method C (deepswabbed method): a self-made 40 -cm long cotton swab was sterilized by an autoclave at $132 \square$; a person held the ends of ventilator pipe, and another one take the sterilized long cotton swab dipping sterile saline to circularly swab the inner wall surface of the threaded pipes at the distal part approximately at the depth of $30 \mathrm{~cm}$ and the swabbed area was not less than $50 \mathrm{~cm}^{2}$; the head of the cotton swabs were clipped with a sterile scissors and placed in the sampling tube for test.

Evaluation Method. Based on the national standards GB15982-2012 "Hospital Disinfection Hygiene Standards", it is qualified in the sterilization that the total number of colonies is $\leq 20$ cfu/piece and no pathogenic microorganism is detected for moderate risk medical devices.

Statistical Methods. The data were analyzed with software SPSS18.0 for statistically and compared with $\chi 2$ test. $P<0.01$ was considered statistically significant.

\section{Results}

As shown in Table 1, the qualified rate of ventilator pipes sterilized with method $\mathrm{C}$ was lower than that with method $\mathrm{B}$ and $\mathrm{C}$ in the same group; taking the results of deep-swabbed sampling method as the criteria, qualified rates of the pipes sterilized with both automatic washing machine and mechanical thermal disinfection in group B were higher than those sterilized with both manual cleaning and immersion disinfection of strongly acidic ionized water in group $\mathrm{A}(P<0.01)$, but the results with the shallow-swabbed or lavage sampling methods were not significantly different. 
Table 1, Comparison on the results with different sampling

\begin{tabular}{cccccc}
\multicolumn{5}{c}{ methods between two groups } \\
\hline groups & \multicolumn{3}{c}{ qualified rates (\%) } & $\chi^{2}$ values & pvalues \\
& method A & method B & method C & & \\
\hline $\mathrm{A}(n=100)$ & 98.32 & 98.32 & 76.22 & 14.18 & 0.001 \\
$\mathrm{~B}(n=100)$ & 100.00 & 100.00 & 92.67 & 5.26 & 0.036 \\
$\chi^{2}$ & 1.03 & 1.03 & 4.52 & & \\
$P$ & 0.45 & 0.45 & 0.002 & & \\
\hline
\end{tabular}

\section{Discussion}

In hospital-acquired infections, the lower respiratory tract infection has become one of the most common infections, and something wrong with the mechanical ventilation is one of the main causes of lower respiratory tract infections[4]. And mechanically assisted ventilation is an important method for the respiratory support in patients with severe respiratory failure. Ventilator pipes are medical devices that directly contact with the organ mucosa of patients and must be disinfected at a high level before use, otherwise, the non-strict disinfection of them may cause ventilator-associated pneumonia, increase the mortality and economic burden of the patients, even lead to unnecessary medical disputes. It is noteworthy that the ventilator threaded pipe is narrow and damp, and most suitable for the growth of microorganisms. The monitoring on the disinfection effect of ventilator pipe is an important approach to evaluate the quality of disinfection. At present, there is no unified domestic sterilization monitoring standards for the disinfection of ventilator pipe system. Beijing "Ventilator Cleaning and Disinfection Guidelines" has recommended a sampling method based on the object surface of disinfecting technical specifications. The sample is collected by swabbing the shallow part of the pipes with a sterile cotton swab according to this sampling method, but as a result, the negative rate is higher, suggesting that it can not truly reflect the actual disinfection effect of the deep part of pipes; the sampling at the deep part of pipe lumen that cotton swabs can not reach is conducted almost by the sterile saline lavage, but the special nature of the lumen wall structure may bring a certain degree of difficulty to the sampling, for example, the lavage solution can dilute the bacteria in the threaded pipe due to the repeated shaking of the pipe, resulting in a difficulty to collect positive samples efficiently, thereby easily covering up the real disinfection situation. The part that it is most difficult for the lavage solution to reach is just the middle part of a threaded pipe with a length equal to or more than $60 \mathrm{~cm} \mathrm{[5].}$

Reuse ventilator pipe is one of more expensive medical equipments. It is difficult to implement the sampling by putting the whole equipment in a sterile tube or destructing the equipment into pieces to sample according to medical equipment disinfection monitoring methods proposed by "Hospital Disinfection Hygiene Standards"[6]. However, it is necessary for us to monitor the disinfecting effect of the reuse ventilator pipe. In this study, a sampling method with a special selfmade long cotton swab that could reach to the middle part of the pipes was used to take the samples at the most difficultly cleaning part of the ventilator pipes for monitoring the disinfection effect of ventilator pipes, during which we could even visualize the dirt that remained on the inner wall adhered to the swab, indicating that the data achieved with this method could objectively reflect the disinfection effect of the deep part of ventilator pipes[7]. The deep-swabbed method can be used to repeatedly swab the deep part of ventilator pipes, which is most difficult to be cleaned and disinfected, to avoid the limitations of the other two sampling methods, and the evaluation with this method should be effective, objective, truthful and reliable, although the performance of this method is difficult, the cotton swab made specially is too long with a length of $40 \mathrm{~cm}$ and necessary to be sterilized, and the operation of the cotton swab needs to be completed by two people's tacit cooperation, indicating that it is worth being popularized.

Ventilator pipe is a moderate dangerous article indirectly contacting with patients mucous membranes, but the invasive application of ventilator for ventilation has shown a high probability in patients, and the contamination of ventilator pipe is an important factor to induce ventilatorassociated pneumonia in patients with a low resistance, so that how to improve the disinfection of 
ventilator pipe has become a hot topic[8]. Because of its unique structure, such as the thread-like wall, long lumen, small diameter, silica gel with a certain elasticity and great friction coefficient, it is difficult for ventilator to be cleaned, especially with a poor cleaning and disinfecting effect at the deep part of it; the qualified disinfection rate of ventilator pipe is lower often due to lack of effective cleaning tools and cleaning means if it is cleaned manually, which has been confirmed by using the deep-swabbed sampling method. The disinfection with an automatic cleaning and disinfecting machine is a high-level disinfection process, in which the lumen of threaded pipe is repeatedly cleaned fully with non-circulating purified water through a special interface and its outer wall is washed by an automatic spray arm to improve the cleaning and disinfection effect[9]. The whole cleaning and disinfecting process is carried out in a closed environment and in accordance with the set procedures. It shows advantages that the threaded pipe of ventilator is not susceptible to the contamination of external environment, and vice versa, and the whole cleaning, disinfecting and drying process is completed strictly in accordance with the procedures, to avoid the nonstandardization and touching contaminants due to the manual operation, the splashing of dirty water, and the harm of chlorine disinfectant to the operators, maximally limiting the risk of occupational exposure[10].

Currently, ventilator pipes are sterilized with automatic washing machine by the disinfection supply centers in hospitals higher than three-class hospital in China and the disinfection effect is more reliable. However, in some small hospitals, ventilator pipes are mostly washed by hand and then dried in the air or with stove dry after they are soaked in chemical disinfectants. But we believe that no matter what methods are used for the disinfection of ventilator pipes, their disinfection effects must be verified to ensure the reliability of disinfection method. The choice of disinfection method should be based on the reliability of disinfection effect, environmental protection, economy, non-toxicity and harmlessness.

\section{References}

[1] Shaoling Wu and Yan Lu: Comparison on effect of different disinfection methods for ventilator tube disinfection. Chinese General Practice Nursing Vol. 11(2013), p. 1121.

[2] Lei Lu and Limei Wang: Comparison on effects of three sampling methods for ventilator pipe disinfection. Chinese Journal of Disinfection Vol. 31(2014), p. 865.

[3] Minru Liu and Bo Duan: Comparative analysis on effects of two different disinfection methods for ventilator pipe. Shanxi Medical Journal Vol. 41( 2012), p. 915.

[4] Qiao Wu and Suwei Du: Evaluation on the effect of acidic electrolyzed oxidized water for the disinfection of ventilator pipe. Chinese Journal of Disinfection Vol. 31(2014), p. 782.

[5] Xiujuan Yin, Yuqin Shen, and Lizhu Xiao: Continuous quality improvement for Ventilator Pipes's accessories processing procedure in the disinfection supply center. Nursing and Rehabilitation Journal Vol.10(2011), p. 814.

[6] Yunxia Jia, and Yinglin Wang: Comparative study on acidic electrolyzed oxidized water in the disinfection of ventilator pipe. Chinese Journal of Nosocomiology Vol. 20(2010), p. 2081.

[7] Chao Chen and Ying Guo: Clinical application of tube washer-disinfector in disinfection of breathing apparatus tube. Chinese Journal of Nosocomiology Vol. 21(2011), p. 125.

[8] Yumei Si: Application of acidic electrolyzed water in disinfection supply center. Chinese Nursing Research Vol. 3(2011), p. 709-710.

[9] Xiaoli Li and Junxia Wang: Discussion on the centralized management mode for ventilator pipes. Chinese Journal of Nosocomiology Vol. 21(2011), p. 4775.

[10] Yingying Liang: Vetilator-associated pneumonia in ICU: Its risk factors and nursing. Chinese Journal of Nosocomiology Vol. 20(2010), p. 799. 